\title{
First Aid Education Should be Expanded to Support the Learner to Develop Both the Skill and the Will to Help
}

\author{
Eric M. Rottenberg, AAS
}

Correspondence:

Eric M. Rottenberg, AAS

485 Harley Drive Apt 414

Columbus, Ohio 43202-1836 USA

E-mail: rottenberg.1@osu.edu

Conflicts of interest: none

Keywords: education; first aid; laypersons

Received: February 28,

Accepted: May 6, 2018

Online publication: July 23, 2018

doi:10.1017/S1049023X18000614
Rottenberg EM. First aid education should be expanded to support the learner to develop both the skill and the will to help. Prehosp Disaster Med. 2018;33(4):454-455.

The majority of active-shooter crime scenes are not deemed to be safe for medical responder entry until long after the violence has occurred; therefore, bystanders need to be capable of rendering emergency first aid in order to save lives. In response to this problem, Ross, et al studied the effect of brief hemorrhage-control education on laypersons' willingness to respond during a traumatic medical emergency. ${ }^{1}$ They found that a short, educational intervention can improve laypersons' self-efficacy and reported willingness to use a tourniquet in an emergency. However, they suggested that identified barriers to act should be addressed when designing future hemorrhage-control public health education campaigns.

Oliver, et al conducted a pilot study to assess when first aid training can encourage an individual's propensity to act in an emergency situation. ${ }^{2}$ It was concluded that activities which allow the learner to explore and discuss behavior in an emergency situation can effectively increase the learner's propensity to act. It was suggested that first aid education should be expanded to support the learner to develop both the skill and the will to help. However, they acknowledged that a major weakness of their study was that it did not include a randomized deception trial. Van de Velde, et al conducted a randomized controlled trial in which after 24 hours of conventional first aid training, the participants either attended an experimental lesson to reduce barriers to helping or they followed a control lesson. ${ }^{3}$ The deception test - which included the study participant, two actors who played the role of passive bystanders, and a third actor who played the role of imminent victim was used to measure the time between the start of the unannounced, simulated emergency and seeking help behavior, along with the number of particular helping actions. They found that supplementary training on dealing with barriers to helping did not alter the helping behavior or increase the speed of intervention. Unfortunately, the deception test evaluated the helping behavior for a heart attack emergency, which does not require any hands-on skills such as would be needed to stop bleeding.

Shotland and Heinold, which was cited and discussed by the authors, conducted a deception trial evaluating the helping response for arterial bleeding after participants obtained American Red Cross (Washington, DC USA) certification in advanced first aid and emergency care, which included training on severe bleeding, poisoning, skeletal injuries, pulmonary and cardiac arrest, and so on. ${ }^{4}$ Although training did not raise the intervention rate, it did dramatically change the effectiveness of the help used and could yield as many as 28 more saved lives out of 80 such incidents. Future deception trials such as this should compare the effect of brief hemorrhage-control education versus the effect of brief hemorrhage-control education plus supplementary training on dealing with barriers specific to helping victims with traumatic hemorrhage (ie, gunshot wound) on willingness to use a tourniquet or other measures such as applying direct pressure. 
References

1. Ross EM, Redman TT, Mapp JG, et al. Stop the Bleed: the effect of hemorrhage control education on laypersons' willingness to respond during a traumatic medical emergency. Prehosp Disaster Med. 2018;33(2):127-132.

2. Oliver E, Cooper J, McKinney D. Can first aid training encourage individuals propensity to act in an emergency situation? A pilot study. Emerg Med J. 2014;31 (6):518-520.
3. Van de Velde S, Roex A, Vangronsveld K, et al. Can training improve laypersons helping behaviour in first aid? A randomised controlled deception trial. Emerg Med J. 2013;30(4):292-297.

4. Shotland RL, Heinold WD. Bystander response to arterial bleeding: helping skills, the decision-making process, and differentiating the helping response. J Pers Soc Psychol. 1985;49(2):347-356. 